Department of Ophthalmology, Division of Infectious Diseases, Tropical Medicine and AIDS, Academic Medical Centre, University of Amsterdam,

Netherlands G J van den Horn C Meenken

M D de Smet

Department of Internal Medicine, Division of Infectious Diseases, Tropical Medicine and AIDS, Academic Medical Centre, University of Amsterdam, Netherlands

S A Danner

P Reiss

Correspondence to: $G \mathrm{~J}$ van den Horn, MD, University of Amsterdam, Academic Medical Centre, Department of

Ophthalmology Rm G2-254, PO Box 22700, $1100 \mathrm{DE}$ Amsterdam, Netherlands.

Accepted for publication 4 March 1998

\title{
Effects of protease inhibitors on the course of CMV retinitis in relation to CD4+ lymphocyte responses in HIV+ patients
}

Gerardus J van den Horn, Christina Meenken, Sven A Danner, Peter Reiss, Marc D de Smet

\begin{abstract}
Aim-To gain insight into the course of CMV retinitis (CMVR) in AIDS patients receiving protease inhibitors $(P I)$, and to evaluate whether certain patterns of CD4 response are indicative of the clinical outcome and the risk of recurrence.

Methods-15 consecutive AIDS patients receiving maintenance therapy for CMVR were included in a prospective observational cohort study at the university hospital between July and October 1996. Patients were evaluated for signs of CMVR activity and intraocular inflammation. CMVR recurrence was defined as the primary clinical endpoint. Follow up was performed until July 1997. No patient was lost to follow up. Clinical outcome was related to $\mathrm{CD} 4+$ lymphocyte counts, which were monitored every 6 weeks. Highly active antiretroviral treatment regimen including PI was started at study entry.

Results-All recurrences $(n=7)$ were in patients who failed to have a sustained increase in CD4 counts, whereas CMVR remained inactive during a follow up of 42-52 weeks in those who were able permanently to restore their CD4 values to $100 \times 10^{6} / 1$ or more $(n=5)$. The remaining three patients died after 12, 16, and 50 weeks, respectively, without recurrences. All relapses of CMVR were seen after 6-16 weeks, and at CD4 counts well below $100 \times 10^{6} / 1$.

Conclusions-The beneficial effects of PI treatment correlate with the pattern of CD4 response. Sustained increases in CD4 counts achieved in the first 16 weeks of treatment are associated with a prolonged period of CMVR quiescence. Poor initial response is associated with a high risk of CMVR recurrence.

(Br f Ophthalmol 1998;82:988-990)
\end{abstract}

Cytomegalovirus retinitis (CMVR) is the most frequent opportunistic eye infection in patients with HIV infection. It is commonly seen in patients with very low CD4+ lymphocyte counts (below $50 \times 10^{6} / 1$ ). Areas of necrotising retinitis, often with extensive intraretinal haemorrhages, are observed, while inflammatory reaction in the anterior segment and the vitreous cavity is usually mild or virtually absent. This lack of reaction is attributed to the inability to mount sufficient immunological responses. Therapy consists of lifelong antiCMV regimens. In spite of therapy, recurrences are common.

Protease inhibitors (PI) may change this classic picture. Impressive results have been reported with triple drug combinations leading to declining plasma HIV-RNA levels below the detection limit of currently available assays in over $90 \%$ of individuals. ${ }^{12}$ Patients with opportunistic infections have sometimes shown prolonged remissions or improvement in the clinical manifestations of their diseases following initiation of PI treatment. ${ }^{3-7}$ Simultaneously, substantially increased CD4 counts have been observed. These observations reflect at least a partial restoration of immune function in patients receiving PI. However, improvement in immune function may also have disadvantageous effects: enhanced inflammatory activity has been observed shortly after installation of PI therapy. ${ }^{8}$

PI have also had a significant impact on CMVR. The overall incidence of CMVR has dramatically decreased. Recurrence free intervals have been prolonged in some individuals to the point where the role of maintenance therapy has been brought into question. However, CMVR has also been observed in patients on PI therapy at CD4 levels higher than previously reported. ${ }^{9}$ With the advent of PI, it has become necessary to re-evaluate our screening and therapeutic approaches with respect to CMVR.

To gain insight into the course of CMVR in patients receiving PI, we carried out a prospective observational study in AIDS patients on maintenance therapy for CMVR in whom PI treatment was initiated. By relating the course of CMVR to the CD4 response over time we attempt to evaluate whether certain patterns of CD4 response are indicative of the clinical outcome and the risk of recurrence. 
Table 1 Patient characteristics before and during protease inhibitor (PI) treatment.

\begin{tabular}{|c|c|c|c|c|c|}
\hline \multirow[b]{2}{*}{ Patient } & \multicolumn{2}{|c|}{ Before PI treatment } & \multicolumn{2}{|c|}{ During PI treatment } & \multirow[b]{2}{*}{ Follow up (weeks) } \\
\hline & $\begin{array}{l}\text { Duration } \\
\text { of CMVR } \\
\text { (weeks) }\end{array}$ & $\begin{array}{l}\text { No of } \\
\text { induction } \\
\text { courses }\end{array}$ & $\begin{array}{l}\text { Time to } \\
\text { progression } \\
\text { (weeks) }\end{array}$ & $\begin{array}{l}\text { Recurrence } \\
\text { (weeks) }\end{array}$ & \\
\hline 1 & 5 & 1 & 10 & Yes & \\
\hline 2 & 9 & 1 & - & No & Died at 12 weeks \\
\hline 3 & 34 & 4 & 16 & Yes & \\
\hline 4 & 40 & 1 & - & No & Died at 50 weeks \\
\hline 5 & 44 & 3 & 10 & Yes & \\
\hline 6 & 2 & 1 & - & No & 52 \\
\hline 7 & 2 & 1 & 8 & Yes & \\
\hline 8 & 8 & 1 & 12 & Yes & \\
\hline 9 & 42 & 3 & 6 & Yes & \\
\hline 10 & 12 & 1 & 8 & Yes & \\
\hline 11 & 54 & 3 & - & No & 52 \\
\hline 12 & 26 & 3 & - & No & 48 \\
\hline 13 & 4 & 1 & - & No & Died at 16 weeks \\
\hline 14 & 2 & 1 & - & No & 42 \\
\hline 15 & 2 & 1 & - & No & 52 \\
\hline
\end{tabular}

\section{Patients and methods}

Between July and October 1996, highly active retroviral treatment including PI was started in 15 consecutive AIDS patients receiving maintenance therapy for CMVR with either ganciclovir or foscarnet. CMVR was diagnosed on clinical grounds (that is, a characteristic funduscopic appearance and a favourable response to anti-CMV therapy) 2-54 weeks before onset of PI treatment. At the time of inclusion, several of the patients had required one or more reinduction courses because of CMVR progression (Table 1). CD4+ lymphocyte counts were determined at onset of PI therapy, and at 6-8 week intervals. Ophthalmological examination was performed every 2 weeks during the first 2 months, and every 4 weeks thereafter, and was focused on two particular aspects-inflammatory signs as an indicator of the patient's ability to mount an inflammatory response and CMVR activity. The inflammatory reaction in the anterior and posterior segments was scored by slit lamp examination as either regular or excessive; CMVR activity was graded funduscopically as completely inactive, smouldering, or active. In

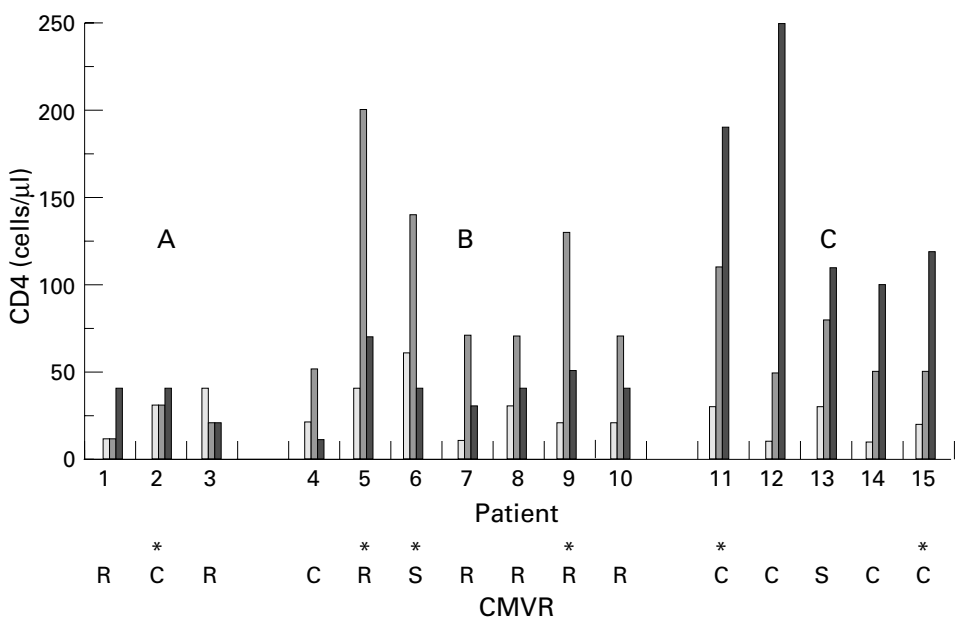

Figure 1 Top: $C D 4$ responses to protease inhibitor (PI) treatment. Individual counts are given at baseline, at 6-8, and at 12-16 weeks. (A) Non-responders. (B) Biphasic responders: an initial increase is followed by a return back to initial values. (C) Sustained responders: after an initial increase, a higher level is reached at 16 weeks and maintained for a prolonged period (up to 52 weeks). Bottom: Outcome with respect to CMVR: $R=$ recurrence; $S=$ smouldering; $C=$ completely inactive. Patient 13 , whose retinitis remained smouldering, was non-compliant with respect to his PI and anti-CMV medications, and died after 16 weeks of follow up. Vitritis is indicated by an asterisk. case of active or smouldering lesions, fundus photographs were helpful to assess retinitis control. All assessments were carried out by two ophthalmologists experienced in AIDS related pathology. The clinical end point was defined by recurrence of CMVR. Recurrences were diagnosed if the borders of any lesion had advanced by more than half an optic disc diameter $(750 \mu \mathrm{m})$, or if any new lesion appeared in a previously unaffected area, compared with baseline conditions. Time of initiation of PI was regarded as baseline.

\section{Results}

CD4 COUNTS

All patients invariably had very low CD4 counts at study onset (mean CD4 $25 \times 10^{6} / 1$, range 10-60). Figure 1 shows the individual CD4 responses to PI treatment of all CMVR patients. Three did not show any increase in their CD4 count after 8 weeks of treatment (non-responders). Seven patients had a rapid increase to values of $50 \times 10^{6} / 1$ or more, followed by a return to nearly initial values over the following 6 weeks. A group of five patients showed a sustained increase in their CD4 counts, and reached values of $100 \times 10^{6} / 1$ or more after 12-16 weeks of treatment. The elevated levels were maintained during the whole follow up period.

\section{VITRITIS}

Six patients showed excessive inflammation in the vitreous cavity $6-8$ weeks after onset of PI medication. They are indicated in Figure 1 by an asterisk. Four of them (nos 5, 6, 9, 11) had a concomitant rapid increase of their CD4 count to values above $100 \times 10^{6} / 1$. Patients experienced blurred vision and floaters, but none required a vitrectomy. Vitritis never interfered with funduscopic assessment.

\section{CMVR}

While using PI, seven patients needed reinduction because of CMVR reactivation. In two patients, CMVR remained smouldering, whereas it became completely inactive in six. CMVR activity in relation to the response to PI treatment is indicated in Figure 1 (bottom). All recurrences were in patients who failed to have sustained CD4 responses to PI treatment, whereas CMVR became inactive in those who were able to permanently restore their CD4 values to $100 \times 10^{6} / 1$ or more. All relapses of CMVR were seen at CD4 counts well below $100 \times 10^{6} / 1$.

\section{Discussion}

This study demonstrates two important phenomena. Firstly, there exists a clear difference in clinical outcome between patients with and without a sustained CD4 response to PI treatment. During a follow up of 42-52 weeks none of the five patients with a sustained response relapsed, whereas seven of 10 patients without a sustained response needed reinduction therapy because of reactivation of CMVR. All recurrences occurred within 4 months. This 
suggests that patients without sustained CD4 response do not benefit from PI with respect to CMVR.

The second phenomenon is that the manifestations of successful PI treatment in severely immunocompromised AIDS patients can be divided into two episodes: an early phase of approximately 6 weeks' duration in which CD4 cells may rise substantially and in which the ability to mount an inflammatory response is enhanced. In this phase, inflammatory reactions such as vitritis may occur. This episode is followed by either a return of CD4 counts to baseline values or by further immune restoration during which CD4 counts remain elevated (sustained response). A return of CD4 counts to baseline conditions preceded a relapse of CMVR in five cases and seems to be indicative of imminent recurrence.

Shortly after initiation of PI treatment we observed vitritis in six patients. This phenomenon has not been previously associated with AIDS related CMVR. It probably reflects an increased inflammatory response to ocular CMV proteins resulting from increased immunocompetence due to PI treatment. It is associated in our data with an early increase of CD4 to values above $100 \times 10^{6} / 1$, although the presence of inflammation is not predictive of a sustained increase in CD4 count. In a recent paper Jacobson et $a l^{9}$ report five cases of CMVR with CD 4 counts around $200 \times 10^{6} / 1$ at the time of CMVR diagnosis. These patients had started PI 4-7 weeks earlier while their CD4 count was less than $85 \times 10^{6} / 1$. Two of these patients also had vitritis. In our opinion, the apparent discrepancy between the diagnosis of CMVR and the relatively high CD4 counts in these patients is a consequence of the fact that in these patients a previously subclinical CMVR progressed to symptomatic disease, while CD4 counts rose simultaneously as a result of initiation of PI treatment. We believe that their observation that no recurrences of CMVR were seen during a follow up of 5-12 months is of greater importance. It is consistent with the lack of progression of CMVR as seen in our patients with sustained CD4 responses. It suggests that immune restoration can be achieved to an extent sufficient to prevent recurrence of CMVR. An important question, which should be addressed in future studies, is whether continued anti-CMV maintenance therapy is still mandatory in patients with a sustained increase in CD4. Another issue worth investigating is whether declining CD4 levels in patients who have previously experienced a sustained increase in CD4 should be regarded as indicative of imminent recurrence. Such studies should not only involve frequent ocular examinations but also include CD4 monitoring in association with other prognostic markers such as determination of CMV-DNA levels in the peripheral blood by polymerase chain reaction. ${ }^{10-12}$

1 Notermans DW, de Wolf F, Foudraine NA, et al. The effects of an antiretroviral triple combination with ritonavir, AZT, and 3-TC. Third International Congress on Drug Therapy in HIV infection. Birmingham, UK, 1996. AIDS 1996;10 in HIV infection. Birmingham,

2 Gulick RM, Mellors J, Havlir D, et al. Potent and sustained antiretroviral activity of indinavir, zidovudine and lamivudine. XIth International Conference on AIDS. Vancouver, 1996 (abstract Th.B.931)

3 Elliot B, Aromin I, Gold R, et al. 2.5 Year remission of AIDS associated progressive multifocal leukencephalopathy with combined antiretroviral therapy. Lancet 1997;349:850.

4 Power C, Nath A, Aoki FY, et al. Remission of progressive multifocal leukoencephalopathy following splenectomy and
antiretroviral therapy in a patient with HIV infection. $N$ Engl f Med 1997;336:661-2.

5 Murphy M, Armstrong D, Sephowitz KA, et al. Regression of AIDS-related Kaposi's sarcoma following treatment with a HIV-1 protease inhibitor. AIDS 1997;11:261-2.

6 Carr A, Foudraine N, Reiss P, et al. Resolution of antibioticresistant cryptosporidiosis and microsporidiosis with potent combination antiretroviral therapy. 4 th Conference on Retroviruses and Opportunistic Infections, Washington, 1997 (abstract 688)

7 Benhamou Y, Bochet MV, Carriere J, et al. Effects of triple antiretroviral therapy including an HIV protease inhibitor on chronic intestinal cryptosporidiosis and microsporidiosis in HIV-infected patients. 4th Conference on Retroviruses and Opportunistic Infections, Washington, 1997 (abstract 357)

8 Carr A, Cooper DA. Restoration of immunity to chronic hepatitis B infection in HIV infected patients on protease inhibitor. Lancet 1997;349:995-6.

9 Jacobson MA, Zegans M, Pavan PR, et al. Cytomegalovirus retinitis after initiation of highly active antiretroviral therapy. Lancet 1997;349:1443-5.

10 Rasmussen L, Morris S, Zipeto D, et al. Quantitation of human cytomegalovirus DNA from peripheral blood cells of human immunodeficiency virus-infected patients could of human immunodeficiency virus-infected patients could
predict cytomegalovirus retinitis. F Infect Dis 1995;171: predict $177-82$.

11 Spector SA, Picher M, Lamy P, et al. PCR of plasma for cytomegalovirus DNA identifies HIV-infected persons most likely to benefit from oral ganciclovir prophylaxis. XIth International Conference on AIDS. Vancouver, 1996 (abstract Th.B. 302)

2 Dodt KK, Jacobsen PH, Hofman B, et al. Development of cytomegalovirus (CMV) disease may be predicted in HIVinfected patients by CMV polymerase chain reaction and the antigenemia test. AIDS 1997;11:21-8. 